\title{
Der Schliisselbeinbruch in der Lnfallversicherung.
}

\author{
Von \\ Prof. Dr. Hans Liniger und Oberstabsarzt a. 1). Dr. Hermann Weber \\ zu Frankfurt a. M.
}

(Eingegangen am 12. Juni 1919.)

Der Schlïsselbeinbruch ist einer der am häufigsten vorkommenden Knochenbrüche. Malgaigne fand ihn unter 2358 Knochenbrüchen 228 mal, also in etwa 10\%. Bardenheuer gibt sein Vorkommen auf $13 \%$, Gurlt, Schreiber und Helferich anf $15 \%$ : Hoffa anf $15-16 \%$ und Pitha auf $18,7 \%$ an. Auf Grund unserer eigenen Erfahrungen halten wir die höheren Prozentsütze für richtig, zumal da viele Schlïsselbeinbrüche, bcsonders bei Kindern glatt übersehen werden. Bei Kindern unter zehn Jahren ist ja der Schlïsselbeinbruch der hauptsächlichst beobachtete Bruch. Die entsprechende Verletzung des Erwachsenen ist die Schultergelenksausrenkung bzw. der typische Speichenbruch. In der Kindheit ist der Schlüsselbeinbruch gleich häufig bei beiden Geschlechtern. Später überwiegt er bei dem männlichen Geschlechte, da der Mann als Arbeiter höheren Gefahren ausgesetzt ist als die Frau. Malgaigne fand nur 58 Briiche bei Frauen gegen 178 bei Yinnem. Die Schlïsselbeinbrüche treten auf beiden Körperseiten gleichmäßig auf. Doppelseitige Schlïsselbeinbrüche sind selten.

Was den Sitz der Bruchstellen anbetrifft, so ist folgendes zu bemerken: Das Schlüsselbein ist S-förmig gekrümmt. Die Krümmung ist in den beiden inneren Dritteln nach vome konvex, $1 \mathrm{~m}$ äuBeren Drittel nach vome konkav. Der Halbmesser der ersten Krüınmung übertrifft jenen der zweiten. Je anhaltender der Arm zu schwerer Arbeit gebraucht wird, desto stärker wird das Schlüsselbein und desto mehr prägt sich seine S-förmige Krümmung aus. An demjenigen Punkte, wo die beiden Krümmungen zusammenstoßen, sitzt die Mehrzahl der Briche. Dieser Punlit ist nach Hyrtl gleichsam der Knotenpunkt, in dem sich die dem Schlüsselbein durch Stoß und Cregenstoß mitgeteilten Schwingungen treffen.

Die Schlüsselbeinbrüche entstehen entweder durch direkte Gewalteinwirkung wie Stoß, Schlag, Auffallen eines Cregenstandes, oder, und das ist das häufigere, indirekt durch Fall auf die Schulter oder Fall auf die Hand bai fixiertem Fllbogen- und Schultergelenk. Dabei wird der Knochen in seiner Längsrichtung gebogen und zum Einknicken gebracht. Auch ein Ablnicken 
des Schlüsselheines an der Stelle, wo es bei stark herabhängender Schulter die erste Rippe berihrt, limmit nach Helferich danu ror, wenn beim Heben schwerer Lasten der Arm stark nich albwärts gerogen wird. Schlüsselbeinbrüche, lediglich durch Honskelzum entstanden. sind selten. Sie lassen meist an Syphilis, Tumoren und Nervenlaranlkeiten denken, doch sollen sie nach Gurlt und Helferich auch bei normalen Knochen vorkommen. Wir haben einen derartigen Fall bisher nicht bcobachtet. Chassaignac berichtet über einen solchen Fall, wo sich ein gesunder Taglöhner beim dufladen von Steinen nur durch Muskelzug einen Schlïsselbeinbruch zugezogen hatte. Un rollständige Brüche, sogenannte Infraktionen, werden vorwiegend bei Kindern beobachtet.

Die Verschiebung der Bruchstiicke hängt hauptsächlich von der Richtung der brechenden Gewalt und des Bruches selbst ab. Die Bruchfläche verläuft gewöhnlich schrägr von außen vorne nach innew hinten. Das äußere Bruchstück wird durch den Zug des Muscuhs deltoideus und subclavins nach unten, das innere durch den Kopfnicker nach oben verschoben, der Arm mit der Schulter, seines Strebepfeilers beraubt, sinkt tiefer, rückt näher an den Brustkorb heran und wird gleichzeitig etwas nach vorne und einwärts geclreht.

Der Brueh libt sich infolge der Beweglichkeit der Bruchstiteke meist leicht einrichten, aber um so schwerer in cler rjchtigen Stellung erhalten. Nicht nur die Atembewegungen, sondern sogar schon Bewegungen der gesunden Schulter äußern ihre Wirhung auf die Bruchstïcke und verursachen leicht eine Verschiebung. Beide Schlüsselbeine hängen durch das Ligamentum interclaviculare untereinander zusammen. So kommt es, daß die besteingerichteten Schlïsselbeinbrüche meist schief verheilen. Die Erhaltung der Bruchstücke in der richtigen Stellung bis zur festen Verheilung golingt nur selten. Daher stammt auch die riesige Zahl der compfohlenen Verbände und Apparate, die sich noch ständig vermehri. Gurlt konnte schon 70 verschiedone Arten auffïhren.

Eine einfache und gute Art der Behandiung hat schon Hippokrates angegeben. Fr ließ Verletzte mit Schlüsselbeinbrüchen mehrere Wochen lang auf dem Rücken liegen, nachdem er zwischen den Schulterblättern ein Kissen befestigt hatte, so daß die Schultem nach hintern sanken. Zweifellos wird hiercurch einer seitlichen Verschiebung der Bruchstücke und somit einer Verkürzung des Schlüsselbeines vorgebeugt.

Pirogoff ließ namentlich bei Kinderm ein eng anschließendes Triliotheund tragen. Die Ärmel der kranken Scite wurden in recht- oder spitzwinkliger Stellung des Ellbogens am Brusteile des Hemdes festgenäht. Dupuytren legte zwei Handuicher an, das eine als Yitella, das andere zirliular zur Fixierung des Oberarmes am Brustkorbe.

Die bekamntesten größeren Ferbände sind die nach Velpeau und Dessault benannten. Die größte Beliebtheit hat sich jedoch bei Schlüselbeinbrïchen der schon über 50 Jahre alte Sayresche Heftpflasterverband mit den bekannten 3 Touren erworben. Das Ideal eines Verbandes stellt aber auch er nicht dar, denn auch bei seiner Anwendung stehen die Bruchstücke oft mehr neben- als aufeinander, besonders wenn etwa das Achselkissen vergessen wurde.

Besondere Erwähnung verdient der Extensionsverband nach Bardenhever, bei welchem der Arm nach hinten oben oder schräg nach außen extendiert wird. Dieser Verband ist für den praktischen Arrt zu kompliziert und daner 
mehr für das Krankenhaus und in diesem auch nur für den clamit eingearbeiteten Facharzt geeignet. Dann zeitigt er aber anch vorzügliche Heilergebnisse, sowohl was gute Stellung der Bruchstücke als auch volle Funktionsfähigkeit des Arnes und des Schultergelenkes anbelangt. Ganz besonders empfiehlt sich das Bardenheuersche Verfahren, wenn aus kosmetischen Gründen, besonders bei Damen, unter allen Umständen eine Entstellung der unteren Halsgegend vermieden werden soll. Kanfmann (Handbuch der Unfallmedizin 1907, S. 340). wendet dieses Verfahren in allen Fälen bei versicherten Arbeitern an. Die Behandlung verlangt 3 Wochen Bettlage.

Cnter Umständen, wenn auch in seltenen Fälen, muß die Naht der Bruchstiucke vorgenommen werden. Sie kann nur in einem Krankenhause und von einem guten Chimrgen ausgeführt werden. Es ist aber hierbei sehr wohl zu beachten, daß bei einem versicherten Unfallverletrten nur im Notfalle eine Operation vorgenommen werden soll, da erfahrungsgemäß in derartigen Fällen die Beschwerden selbst nach gut geglückter Operation oft größer sind als vor dersclhen. Werden gar Drahtnähte an der Bruchstelle des Knochens versenkt, so kann man sicher sein, daß die Beschwerden spaiter größer sein werden, als wemu der Schlüsselbeinbruch sich selbst überlassen geblieben wäre.

Ein besonderes Heilrerfahren ist das sofortige Forgehen mit kunstgerechter Vassage und Bewegungen. Die erzielten Heilergebnisse sind ganz vorzüglich, besonders wenn es sich um ältere Patienten handelt, bei denen das Schultergelenk nicht längere Zeit steif gestellt werden darf. Bei Kindern und jungen Lenten wird die Ruhigstellung des Schultergelenkes dureh Verbinde selbst 3--4 Wochen lang gut vertragen, das Gelenk wird nach relativ kurzer Zeit wiedur funktionsfahig. Anders bei älteren Leuten. Hier führt schon eine 14-tägigo Ruhestellung des Gelenkes oft zu einer derartigen Versteifung, daß in vielen Fällen eine vollkommene Wieclerherstellung der freien Beweglichkeit nicht mehr zu erzielen ist. Es ist deshalb in Fällen von Schlüselbeinbrüchen bei älteren Leuten, etwa vom 50 . Lebensjahre ab, ein Kunstfehler, das nichtverletzte Sichultergelenk für mehrere Wochen steif zu stellen. Dies geschieht aber leider auch noch heutzutage ziemlich häufig. Wir verweisen in dieser Hinsicht auf die Feröfentlichngen von Lucas-Championniere, de Warbaix, Korteweg, Thiem, sowie auf die Arbeit von Liniger ,. Wodeme Bchandlung von Unfallverletzungen in den Jahreskursen für ärtliche Fortbildung": Septemberheft 191\%, S. 26. Eine seltsame Art der Behandlung schlägt Dr. \$. Kof mann in Odessa vor kurzem im Zentraiblatt für Chirurgie vor: Die Bruchstelle wird eingerichtet, die Hand entweder in dit Hittellagehangen oder zwischen den Bocklinöpfen gehalien, damn wird der Arm regelmäßig bewergt. Soweit ist, das Heilverfahren durchans modern und richtig. Nun kommit aber das Merkwïrdige: Kof mann empfiehlt nummehr die unter Winkelstellung der Bruchstücke verheilte Bruchstelle freizulegen und den Knochen mit Hamuner und Heißel möglichst wohl zu formen. Dadurch werde eine ungestörte Armhebung, die vorher durch den Kallus verhindert sei, gewährleistet. Nuch unserex Erfahrung stört der Kallus ïberhumpt nicht und behindert dis Bewegungen im Schultergelenk nicht. Wir halten diese Operation für überflüssig und bei Versicherten geradezu für bedenklich. Wer wird sich überhaupt einen geheilten Sehluisselbeinbruch noch operieren lassen? 
Selbst ohne Behandlung heilen die Schlüsselbeinbrüche fast immer git. Pseudarthrosen sind sehr selten. Wir selbst haben nur 3 einschlägige Fialle beobachtet:

1. 31 jähriger Schlosser. Bruch des linken Schlüsselbeins etwas außerhalb der Nitte durch Aufschlagen eines Balkens. Zunächst 3 Wochen Sayrescher Heftpflaster-, dann noch 3 Wochen lang Gipsverband. Der Bruch wurde überhaupt nicht fest. Nach 2 Jahren Dauerrente von 20\%. Der Verletzte: wurde später abgefunden und arbeitete dann wieder als Schlosser ohne jede Behinderung.

2. 48jähriger Tag]öhner. Fall auf die rechte Schulter 1901. Bruch des Schlüsselbeines an der Grenze von mittlerem und äuBerem Drittel. Alter Luetiker. Nachuntersuchung durch Prof. Liniger 1909: Bruch nicht knöchem verheilt. Keine Versteifung der Schulter. Keine Huskelschwäche. Es wurde eine Danerrente von $15 \%$ rewährt. Der Verletzte arbeitete wieder ohne Lohnausfall.

3. 57jähriger Baumeister. Ater Diabetiker. 1916 Brich des rechten Schlüsselbeines an der Grenze von äußerem und mittlerem Drittel durch Aufschlagen eines Balkens. Untersuchung durch Prof. Liniger 1918. Nicht knöchern verheilt. Der Verletzte behauptet, der Assistenzarzt habe den Verband zu früh, schon nach 3 Wochen entfernt und die Bewegungen zu forciert alsgefïhrt. Schultergelenk frei. Muskulatur an der Schulter leicht gesehwächt, am Arme nicht: Keine objektiven nervösen Störungen. Klagen sehr stark, besonders auch über Gefühlsstörungen im Ulnarisgebiet. Verrichtet aber seinen vollen Dienst. Der rechte Arm wird auf Grund der Versicherungsbedingungen als um ein Drittel geschüdigt bezeichnet, der Verletzte entsprechend abgefunden.

In allen drei Fällen war also die Minderung der Erwerbsfiahigkeit trotz Pseudarthrose nicht wesentlich, wenn auch die betreffenden Verletzten z. T. auf Grund von Entscheidungen der Instanzen, z. T. auf Grund der Versicherungsbeclingungen Entschädigungen erhielten. Die Ansicht Pithas im Handbuch der Alg. u. Spez. Chirurgie ron Billroth, S. 8, daß die Pseudarthrose zumal für den Arbeiter ein trauriges Ereignis sei, das eine pernanente schwere Funktionsstörung mit sich führe, ist nicht zutreffend. Neben unserer Erfahrung führen wir noch folgende Autoren an:

A. Köhler in Eulenburgs Rucalenzyklopädie, Bd. XIII, S. 230: Pseudarthrosen des Schliusselbeins sind äußerst selten beobachtet. Sie stören die Funktion des Armes wenig oder gar nicht.

Silberstein, Lehrbuch der Unfallheilkunde, S. 207: Selbst Pseudarthrosen sind nicht immer funktionsstörend.

Pietrzikowski, Begutachtung der Unfallverletzungen, S. 407: Selbst deutliche Pseudarthrosen hinterlassen in der Regel keine schwere funlitionelle Behinderung.

Gelpke und Sohlatter, Unfallkunde, S. 317: Pseudarthrosen beeinträchtigen nicht immer die Erwerbsfähigkeit.

Sicher ist, daß die Funktionsstörungen nach Pseudarthrose des Schlüsselbeines von vielen Gutachtem weit überschätzt werden, und daß die von $A$ bder- 
halden mitgeteilten Fälle, bei denen die Erwerbsfähigkeit auf $40 \mathrm{bzw} .50 \%$ geschätzt wurde, und ein rom Reichsversicherungsamt entschiedener Fall, der sogar mit einer Rente von $66^{2} / 3 \%$ bedacht wurde, schwere Niebenbefunde aufweisen mußten, z. B. starke Bewegungsstörungen in der Schulter, wesentliche Schwäche des Armes usw. Wesentliche Beeinträchtigung des Armes durch abnorm starke Kallusbildung, durch Verletzungen von Nervenstämmen oder größerer Blutgefäße haben wir nicht beobachtet. Wir verfügen auch über keinen Fall, in dem die Lunge mitbeteiligt gewesen wäre.

Die Prognose der Schlüsselbeinbrüche wird in den Lehrbüchem über Frakturen und Luxationen als sehr günstig bezeichnet. 'Tatsächlich heilen die meisten Schlüsselbeinbrüche, selbst solche, die nach der Heilung erhebliche Verschiebung der Bruchstücke aufweisen, ohne wesentliche Gebrauchsstörung des Armes aus.

Uber die Heilungslauer bei Versicherten geben folgende Statistiken Aıfschluß:

Die Österreichische Unfall-Statistik für die Jahre 1897-1901: Bei 730 Fïllen danerte das Heilverfahren durehschnittlich 47 Tage. $50 \%$ der Fälle mußten aber dauend entschädigt werden.

Erfurth (Festschrift Cottbus) berichtet über die Heilergebnisse in 264 Fïllen. Interessant ist seine Feststellung, daß die Heilergebnisse am besten waren bei ausschlieblicher Krankenhausbehandlung. Es crhielten noch Rente nach thlauf eines Jahres $57,4 \%$, nach 10 . Jahren $1,85 \%$. In 4weiter Linje kamen diejenigen, die anfänglich zu Hause und später im mediko-mechanisohen Institut behandelt wurden. Rente wurde gezahlt nach $1 \mathrm{~J}$ ahre in $60 \%$, nach 10. Jahren in 3.3\%. Dann folgen die nur zu Hause Behandelten mit Rente nach $1 \mathrm{~J}$ ahre in $68,6 \%$, nach 10 . Jahren in $4,65 \%$, und am ungünstigster sind die von Kurpfuschern behandelten Fälle verlaufen Bei ihnen wurde noch Rente gezablt nach 1 . Tahre in $100 \%$, nach 10 Jahren in $14,29 \%$ der Falle. Erfurth betont noch besonders, wie außerordentlich bedeutungsvoll für den Heilverlauf der möglichst frïhzeitige Beginn mit Bewegungsïbungen sei.

Die Winterthurer Statistik bringt 150 Fille von Schlüsselbeinbrüchen. Davon heilten $25 \%$ im Laufe des ersten, $50 \%$ in Lalufe des zweiten Wonats und $25 \%$ brauchten zur Heilung mehr als 9 Wochen. Nir in $4=2,7 \%$ der Fïlle trat Invalidität ein.

Um nun auch die Heilergebnisse. in der privaten Uruall-Forsicherung kenuen zu lernen, haben wir das Archiv der Trankfurter Allgemeinen Versicherungs-Aktien-Gesellschaft daraufhin durchgesehen und fanden unter den letzten 24080 Schadenfïllen die Krankengeschichten von 100 Fällen reiner. d. h. nicht mit anderen Verletzungen einhergehender Schlïsselbeinbrüche. In der Privat-Versicherung spielt der Schlüsselbeinbruch denurach im Verhältnis zu den übrigen Körperschäden eine recht geringe Rolle. Bei der Frankfurter berechnet sich das $\vec{V}$ erhältnis auf $0,1 \% \%$.

In diesen 100 Fällen erfolgte die Wiederherstellung roller Arbeitsfähigkeit durchschnittlich nach 42 Tagen. Volle Erwerbsunfähigheit bestand durchschnittlich nur 20 Tage lang. Während des Restes der Behandlungszeit war die Erwerbsfahigkeit nur teilweise herabgesetzt. Je jünger die Verletaten waren, desto kürzer war im allgemeinen die Behandlungsdaner. 23 unserer Fälle ge- 
hörtern der Altersklasse von 14-20 Jahren an. Bei ihnen war die volle Erwerbsfühigkeit durchschnjttlich bereits nach 20 Tagen wiederhergestellt. 5 von unseren Fällen wiesen eine Behandlungsdauer von ïber 100, ciner sogar eine solche ron 141 Tagen auf. Dieser ungïnstige Verlauf war zum Teil auf unzweckmäßige Behandlung zurückzuführen. Der Arn war zu Jange ruhig gestellt, es war infolge dessen Versteifung des Schultergelenks und Schwäche der Muskulatur eingetreten, die eine lange mediko-mechanische Nachbehandlung erforderich machten. Zum Tejl lag doloses Verhalten des Verletzten vor, der clen Unfall gründlich ausnutzen wollte. Zu ciner danemden Beeinträchtigung der Arbeitsfahigkeit (Invalidität) ist es in keinem einzigen unserer Fälle gekommen.

An Entsehädigung für vorülergehende Arbeitsunfühichlieit hatte die Gesellschaft im Durchschnitt für den einzelnen Fall den 27 fachen Betras der versicherten täglichen Entschädigung zu zahlen. Diese Feststelhng ist für die Berechnung der Reserve sehr wertvoll.

Diejenigen Falle, die der Vertrauensarzt der Berufsgenossonschaft nach Ablauf der 13. Woche zu sehen bekommt, zeichnen sich meistens dadurch aus, daß der Verletzte in höherem Lebensalter stand, oder daß der Bruch in ungünstiger Weise verheilt ist, ocler diß infolge zu langer Puhigstellung im Verbande eine erhebliche Versteifung des Schultergelenks mit Atrophie der Muskulatur eingetreten ist. Würden die Berufsgenossenschaften die Fälle von Schliisselbeinbrüchen bei älteren Personen sofort fachärztlich behandeln oder doch friihzeitig durch einen Fachart liontrollieren lassen, so würle das Heilergebnis sicher ein besseres sein, als es sich $z$. B. aus ctem Statistiken von Erfurth und besonders der österreichischen ergibt. Was fachärztliche Behandlung beim Schlïsselbeinbruch zu leisten vermag, möge folgendes zeigen:

De Marbaix hat Schlïsselbeinbrüche vom 1. Tage ab mit Massage und passiven Bewegungen behandeln lassen. In 4.1 Fällen trat Heilung nach 30 Tagen ein. Kein Fall führte zur Invaliditit. In 22 Fälen wurde die Nassage und Bewegungsbehandlung orst nach 10-15 Tagen aufgenommen. Bis zur Heilung bedurfte es hier schon 95 Tage und ein Fall führte zur Invaliditiat.

Courtault gibt folgende Statistik: 8 Fille ron Schlïsselbeinbrïchen wurden etwa nach 3 Wochen mit Bewegungen behandelt. Die Feildaner betrug 63 Tage. Ein Fall führte zu Invaliditat. In 20 Fïllen wurde mit der Bewegungsbehandlung erst einen Mlonat nach dem Cufall begonmen. Die durchschinittiche Behandlungsdauer betrug 157 Tage. 3 Fälle führten zur Invaliditat.

Prof. Liniger selbst hat während seiner mehrjährigen Tätigleit im Kriege als Leiter des Therapentikums des Städtischen Krankenhauses zu Frankfurt a. M. in 5 Fällen von Schlüsselbeinbrüchen sofort mit Massage und Beweglangen begonnen und den Arm nur in der ersten Woche zeitweise in einem Tragtuche halten lassen. In 3 Fiallen handelte es sich um Kinder, bej denen der Schlüsselbeinbruch mit guter Stellung der Bruchstïcke und wit voller Gebrauchsfähigkeit cles Armes nach 4 Wochen geheilt war. Ein Fall betraf einen 30 jährigen Arbeiter, der nach 6 Wochen wieder voll arbeitsfähig wurde. Im letzten Fall handelte es sich um eine 66 jährige Frau L. aus F., die sich am 7.8 . 17 durch Stur\% von der Treppe einen Bruch des rechten Schlïsselbeins zuzog. Die Frau kam unmittelbar nach dem Unfalle in Behandlung. Es wurcle sofort mit Massage 
und Bewegungen begonnen. Nach der ersten Massage-Sitzung verspiurte die recht ängstliche Frau bereits wesentliche Erleichterung und Nachlassen der Schmerzen. 4 Tage lang wurde der Arm in ein Tragetuch gelegt, das am 5. Tage weggelassen wurde. Die Bewegungen waren nummehr schon fast schmerzlos. Nach 3 Wochen konnte der Arm zu leichten Verrichtungen gebraucht werden. Nach 4 Wochen wurde die Behandlung beendet. Der Bruch war mit der äblichen feichten Stufonbildung fest verheilt, das Schultergelenk frei beweglich, es bestand keine Muskelschwäche. Die Frau hatte zwar noch eine Zeitlang ein gewisses Emnïdungsgefühl im Am, aber keine Schmerzen mehr und konnte? ihre Arbeit im Haushalt wieder voll aufnebmen.

Im Gegensatz zu diesen Falle führen wir 2 Fälle an, in denen nur durch mzweckmäßige Bahandlung ein höchst ungünstiges Heilergebnis erzielt wurde.

Der $36 j$ jahrigo Küfermeister H. aus B. zog sich $\mathrm{sm} 8$. 1. 18 durch Siturz bei Glatteis einen Bruch des linken Schlüsselbeins in der Witte zu. Sayrescher. Heftpflasterverbud 14 Tage lang, darn Dessaultscher Verband 14 'Tage lang, danu Ammtragetuch 3 Wochen lang. Erst dann Bewegungen und Einreibungen durch die Fran des Verletzten. Am 12.4. 18 schreibt der Arzt: Bruch in guter Ferheilung. Bruchstelle leicht empfindlich. Heben des Atrnes etwas ïber die Horizontale möglich. Rückwärtsbewegung noch stark erschwert. $100 \% \mathrm{Er}$ werbsunfähigkeit. Am 6. 5. 18: Schultergelenk noch wesentlich beeinträichtigt. besonders Riickwärtsbewegung. Schmerzen in der Schulter bei Bewegungen, Schwäche des Armes. Mediko-mechanische Behandlung ist eingeleitet. $50 \%$. tro 31. 5. 18: Behandlung abgeschlossen. Bewegungen noch behindert und schmerzhaft. Arm geschwächt. $20 \%$ vorläufig auf $1 / 2$ Jah.r. Ob Dauerschaden eintritt, ist noch nicht sicher. Der Arm wurde nicht mehr voll gebranchsfühig und wurde mit $10 \%$ Invaliditiat abgefunden.

Der 68 jühige Naurer $R$. stürzt am 1. 2. 18 auf verejstem Bürgersteige und bricht das rechte Schlüsselbein etwas außerhalb der Mitte. Haftpflichtfall. Arzt legt sofort fixierten Verband an und berichtet am 17. $5 .: \mathrm{R}$ kann den Arm nicht gebrauchen, da er durch Verhand j Wochen fixiert werden inußte. Vorkiufig ist noch Stcifigkeit der Schulter und des Ellbogengelenks vorhanden. Patient wird mit Massage und Bowegungen behandelt. Er ist noch ganz erwerbsunfähig. Kontrolluntersuchung am 1. 6. 18: Wegen Schulterversteifung und Wuskelschwäche noch völlige Gebrauchsunfähigkeit des Armes. Der Fall wurde wegen zweifellos bestehender Invalidität abgefunden.

Ein dritter Haftpflichtfall ist ebenfalls sehr lehrreich. Der 12 jahrige: Schiiler O. P. zieht sich aru 4. 2. 19 durch Sturz einen Bruch des rechten Schliisselbeins in der Witte zu. Der Arzt schreibt am 20. 5.: Behandlung bestand in Puposition und Sayreschem Verband. Nich Heilung des Bruches Beginn mit Massage und Bewegungen. Die Behandlung wird am 31. 3. 19 beendet. Am 20. 5. ergibt sich folgender Befund: Bruch unter mäßiger Verdickung der Bruchstelle und geringer Verschiebung der Bruchstïcke zueinander verheilt. Keine Druckempfindlichkeit. Bewegungen im Schultergelenk nicht behindert. Urteil: Wesentliche Unfallfolgon sind nicht zurückgeblieben. Die Erwerbsunfähigkeit wird vom 4. 2.-5. 3. anf $100{ }^{\circ}{ }_{0}$ und rom $6 .-31$. 3. auf $50 \%$ geschiitzt.

Die Mehrzahl der Ärzte wird diesen Fall wohl als in jeder Hinsicht normal bezeichnen. Typische Art der Behandlung, glatter Heilverlauf, Heilung inner- 
halb von 2 Honaten. Und doch sind sowohl gegen die Art der Behandlung wie gegen die angenommenen Grade dex Erwerbsunfähigkeit Einwendungen zu machen. Zweifellos hat der Arm zulange im Verbande gelegen. Daher die $100 \%$ bis zum 5. 3. Sehen wir uns unsere Statistik an, so hätte der Fall bis zu diesem Zeitpunkte, ja sogar sehon nach 3 Wochen, geheilt sein können, wenn der Verband nicht zu lange gelegen hätte. Nur ans dicsem Grunde ist auch die verhältnismäßig lange Nachbehandlung bis 31. 3. exforderlich gewesen. Daß der Verband nichts Wesentliches genutzt hat, ist daraus zu ersehen, daß trotzdem die übliche Verschiebung der Bruchstücke eintrat. Nur dem Limstande, daß es sich um einen jungen Nenschen handelt, ist es zuzuschreiben, daß der Fall so relativ günstig verlief.

tber zahlreiche ähnliche fälle könnten wir berichten, bei denen relativ ungünstige Heilergebnisse gezeitigt wurden, und zwar nur aus dem Grunde, weil der Arm, wie es die Schulmedizin verlangt, zunächst wochenlang bis zur kuöchernen Heilung des Schlüsselheinbruches im Verbande ruhig gestellt vurde. Das nicht verletzte Schultergelenk und die unbeteiligte Nuskulatur der Schulter und des Armes litten unter dieser Behandlung ganz erheblich, und später muBten dann die Folgen dieser Behandlung durch längere mediko-mechanische Nachkuren, soweit es ïberhaupt noch möglich war, beseitigt nerden. Dabei ergab sich in fast allen Fällen, daß die Bruchstücke in derselben Winkelstellung, in der sie kurz nach dem Unfalle standen, verheilt waren. Wir raten deshalb dringend auf Grund unserer Erfahrung von der Ruhigstellung der Schulter und des Armes bei Schlüsselbeinbrüchen durch Verbände abzusehen, höchstens den Arm für einige Tage in eine Mitella zu legen, daneben aber sofort mit Massage und Bewegungen zu beginnen. Daß diese Behandlung kunstgerecht ausgeführt werden muB, brauchen wir nicht $z u$ betonen. Es ist Sache der Ärte, sich in dieser Behandlungsmethode auszubilden, resp. die Fälle Fachärzten oder doch gründlich ausgebildeten Masseuren zu überweisen. Wassage durch Angehörige ist Unfug. Bei dieser sogenannten funktionellen Art der Behandlung, die sich, was wir immer wieder betonen müssen, für ältere Verletzte gerarlezu dringend empfiehlt, treten keine Pseudarthrosen ein. Die Heilung des Knochenbruches rrfolgt vielmehr mindestens in der gleichen Zeit und die Brachstücke stehen auch nicht ungünstiger als bei der Anwendung von fixierenden Verbänden. 